\title{
Beneficios de los antibióticos en la rotura prematura de membranas de pretérmino y factores que intervienen en la eficacia del tratamiento. Revisión narrativa
}

\section{Benefits of antibiotics in preterm premature rupture of membranes and factors involved in the efficacy of the treatment. Narrative review}

\author{
Alfredo Ovalle ${ }^{1,2 *}$ y Jorge Figueroa ${ }^{1,2}$
}

${ }^{1}$ Servicio de Obstetricia, Ginecología y Neonatología, Hospital Clínico San Borja Arriarán; ${ }^{2}$ Facultad de Medicina, Universidad de Chile. Santiago, Chile

\section{Resumen}

El parto prematuro es la principal causa de morbilidad y de mortalidad perinatal, y hasta un tercio de los casos presentan rotura prematura de membranas. La infección intrauterina que asciende desde la vagina es su principal causa en un hospital público de Chile. Esta revisión narrativa mediante búsqueda en PubMed, Cochrane, Embase, Scielo, Science Direct y Wiley Online Library incluye estudios publicados sobre los diferentes factores infecciosos que intervienen en el resultado adverso perinatal y la eficacia de los antibióticos en la rotura prematura de membranas de pretérmino. Además, contiene recomendaciones de sociedades científicas sobre el uso de antibióticos en estos casos. Los ensayos concluyen que los antimicrobianos prolongan el embarazo, disminuyen la corioamnionitis clínica y reducen variadas morbilidades neonatales, pero no reducen la mortalidad perinatal ni las secuelas tardías en la infancia. Los resultados adversos obstétricos, especialmente los neonatales, y las secuelas dependen de la existencia de invasión microbiana de la cavidad amniótica o de infección cérvico-vaginal, de la virulencia de los microorganismos aislados, del compromiso inflamatoriolinfeccioso de la placenta (corioamnionitis histológica, funisitis) y de la respuesta inflamatoria fetal. Para mejorar los resultados adversos obstétricos neonatales en la rotura prematura de membranas de pretérmino, los esquemas de antibióticos deben ser eficaces, cubriendo el amplio espectro microbiológico existente y actuando sobre los factores infecciosos implicados en la gravedad de la infección. Además, deben administrarse de manera intensiva y prolongada hasta el parto.

Palabras clave: Rotura prematura de membranas de pretérmino. Terapia antibiótica. Infección intraamniótica. Resultados neonatales.

\section{Abstract}

Preterm birth is the leading cause of perinatal morbidity and mortality, and up to a third of them have premature rupture of membranes. Intrauterine infection that rises from the vagina is its main cause in a public hospital in Chile. This narrative review by searching PubMed, Cochrane, Embase, Scielo, Science Direct and Wiley Online Library includes published studies of the different infectious factors involved in perinatal adverse outcome and of the efficacy of antibiotics in preterm premature rupture of membranes. It also contains recommendations from scientific societies on the use of antibiotics in these cases. These trials conclude that antimicrobials prolong pregnancy, decrease clinical chorioamnionitis, and reduce various neonatal morbidities, but do not reduce perinatal mortality or infant sequelae. Obstetric and especially neonatal adverse outcomes in these patients depend on the existence of microbial invasion of the amniotic cavity and/or cervicovaginal infection, of the virulence of the isolated microorganisms, of inflammatory/infectious involvement of the placenta (histological chorioamnionitis, funisitis) and fetal

*Correspondencia:

*Alfredo Ovalle-Salas

E-mail: alfredoovalle@gmail.com

0048-766X / ๔ 2021 Sociedad Chilena de Obstetricia y Ginecología. Publicado por Permanyer. Éste es un artículo open access bajo la licencia CC BY-NC-ND (https://creativecommons.org/licenses/by-nc-nd/4.0/).

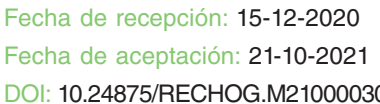

Disponible en internet: 23-12-2021 Rev Chil Obstet Ginecol. 2021;86(5):474-484 www. rechog.com 
A. Ovalle, J. Figueroa: Beneficios de los antibióticos en la rotura prematura de membranas de pretérmino

inflammatory response. To improve adverse neonatal obstetric outcomes in preterm premature rupture of membranes, antibiotic regimens must be effective, covering the wide existing microbiological spectrum and acting on infectious factors responsible for the severity of the infection. In addition, they must be administered aggressively and for a long time until delivery.

Key words: Preterm premature rupture of membranes. Antibiotic therapy. Intra-amniotic infection. Neonatal outcomes.

\section{Introducción}

La tasa de nacimientos prematuros (entre las $22 \mathrm{y}$ 36.6 semanas de gestación) es del $5 \%$ al $9 \%$ de todos los nacimientos en Europa, y del $12 \%$ al $13 \%$ en los Estados Unidos de América. En Chile, en el Hospital Clínico San Borja Arriarán (HCSBA) la tasa de prematuridad fue el $11 \%$ en 2017. Alrededor del $30 \%$ al $35 \%$ de los partos prematuros (PP) son el resultado de una enfermedad materna o fetal, del $40 \%$ al $45 \%$ son consecuencia del trabajo de parto prematuro espontáneo y del $25 \%$ al $30 \%$ de la rotura prematura de membranas (RPM). Una vez que las membranas se rompen prematuramente, el $50 \%$ de las mujeres comenzarán el parto dentro de las 24 a 48 horas siguientes, y del $70 \%$ al $90 \%$ en 7 días. Los niños prematuros están expuestos a una alta mortalidad y morbilidad neonatal. Además, tienen un mayor riesgo de parálisis cerebral y de enfermedad pulmonar crónica, que son más frecuentes en los nacimientos de menor edad gestacional. Las causas de la RPM son multifactoriales, pero la infección que se inicia en la vagina y asciende a la cavidad amniótica (infección bacteriana ascendente) tiene un papel importante en el PP con RPM ${ }^{\dagger}$. En el HCSBA, la infección bacteriana ascendente fue el factor de riesgo más frecuente de PP temprano ${ }^{2}$ y de mortalidad perinatal en este grupo ${ }^{3}$.

La indicación sistemática de antibióticos en las mujeres con RPM de pretérmino (PT), sin trabajo de parto y sin corioamnionitis clínica, prolonga el embarazo y reduce una serie de morbilidades neonatales, pero no disminuye la mortalidad perinatal ni las secuelas a largo plazo ${ }^{1,4}$.

El objetivo de esta revisión narrativa es identificar la asociación antimicrobiana más eficaz para reducir la morbilidad/mortalidad neonatal y las secuelas en la infancia en pacientes con RPM de PT, así como analizar la participación de los factores infecciosos en el resultado adverso perinatal, como la microbiología de la infección intraamniótica y de la infección cérvico-vaginal (ICV) $)^{5}$, la virulencia de los microorganismos aislados $^{6}$ y la infección/inflamación aguda placentaria (corioamnionitis histológica $[\mathrm{CH}]$, funisitis) ${ }^{7}$, y la respuesta inflamatoria fetal ${ }^{8}$. El conocimiento de estas variables permite elegir la asociación antimicrobiana más eficaz para reducir los malos resultados en la RPM de PT.

\section{Revisión narrativa}

\section{Metodología}

En esta revisión narrativa se seleccionaron revisiones sistemáticas, metaanálisis y manuscritos destacados (estudios de cohortes o de casos y controles con riesgo muy bajo o bajo de sesgo y con alta o moderada probabilidad de establecer una relación causal), mediante búsqueda en PubMed, Cochrane, Embase, Scielo, Science Direct y Wiley Online Library, principales fuentes de búsqueda existentes en las Bibliotecas de la Universidad de Chile. Incluye estudios publicados sobre los factores infecciosos implicados en el resultado adverso perinatal y la eficacia de los antibióticos en embarazadas con RPM de PT, desde 1989 en adelante. Además, se presentan las recomendaciones sobre el uso de antibióticos en pacientes con RPM de PT de las principales sociedades y organizaciones médicas de obstetricia y ginecología de países europeos y americanos.

\section{Frecuencia y microorganismos aislados en la invasión microbiana de la cavidad amniótica y la infección cérvico-vaginal en embarazadas con rotura prematura de membranas de pretérmino}

La frecuencia de la invasión microbiana de la cavidad amniótica (IMCA) en pacientes con RPM de PT entre 20 y 35 semanas sin trabajo de parto, utilizando técnicas de cultivo, reacción en cadena de la polimerasa (PCR) más espectrometría de masas por ionización por electropulverización (ESI-MS) y la combinación de cultivo con PCR/ESI-MS fue del $24 \%$, el $36 \%$ y el $41 \%$, respectivamente ${ }^{9}$.

En otra serie ${ }^{10}$, en mujeres con RPM entre 15 y 37 semanas la frecuencia de IMCA por cultivo, PCR y la combinación de estas dos pruebas fue del $34 \%$, el $45 \%$ y el $50 \%$, respectivamente. Los microorganismos 
con más frecuencia encontrados en la cavidad amniótica fueron Sneathia amnii spp. y Ureaplasma urealyticum.

En el HCSBA, en series de embarazadas con RPM entre 24 y 34 semanas, sin trabajo de parto y sin infección clínica, la prevalencia de IMCA fue del $50 \%$ y el $54 \%{ }^{11,12}$. Todos los casos con IMCA tuvieron ICV. La prevalencia de la ICV sin IMCA fue del $24 \%$ y el $22 \%$. Por lo tanto, la frecuencia de pacientes con IMCA e ICV fue del $74 \%$ y el $76 \%$, respectivamente. La IMCA se diagnosticó con cultivo positivo de líquido amniótico obtenido por amniocentesis. Los microorganismos más frecuentemente aislados mediante cultivo (bacterias aerobias, anaerobias y micoplasmas genitales) fueron U. urealyticum, Mycoplasma hominis y Streptococcus agalactiae (estreptococos del grupo B [EGB]), y de las muestras cérvico-vaginales U. urealyticum, Gardnerella vaginalis, M. hominis y SGB. La ICV se diagnosticó cuando se encontró vaginosis bacteriana o vaginitis aeróbica, o micoplasmas genitales, junto con un aumento en el recuento de glóbulos blancos (> 10/ campo $400 \times)^{5}$. La prevalencia de vaginosis bacteriana fue del $51 \%$ y el $48 \%{ }^{11,12}$. El diagnóstico microbiológico de vaginosis bacteriana se hizo mediante la evaluación de la tinción de Gram del flujo vaginal según el método propuesto por Nugent et al para el diagnóstico de VB, está validado en microbiología y habitualmente no se coloca la referencia, los ureaplasmas figuran como $U$. urealyticum, en nomenclatura antigua ${ }^{13}$. Desde 1993, $U$. urealyticum se caracterizó en dos cepas con propiedades antigénicas diferentes: Ureaplasma parvum y Ureaplasma urealyticum ${ }^{13}$.

\section{Frecuencia de la corioamnionitis histológica/funisitis aguda en la rotura prematura de membranas de pretérmino. Relación con la infección cérvicovaginall invasión microbiana de la cavidad amniótica}

$\mathrm{La} \mathrm{CH}$ se encontró en el $58 \%$ y la funisitis en el $37 \%$ de las placentas con RPM de $\mathrm{PT}^{7}$. La funisitis se presentó siempre asociada con $\mathrm{CH}$. Ambas lesiones placentarias son hallazgos patognomónicos de infección. En una serie de PP con RPM ${ }^{6}$, las pacientes con IMCA por SGB, Fusobacterium nucleatum o Haemophilus influenzae, o con IMCA por otras bacterias únicas o asociadas, tuvieron con más frecuencia $\mathrm{CH}(100 \%$ y $88 \%$ ) y funisitis (64\% y $80 \%)$, respectivamente, que las pacientes sin IMCA y sin ICV (17\% y $4 \%)(p<0,001)^{6}$. En otra serie ${ }^{7}$, un estudio prospectivo de 71 pacientes con RPM de PT entre 24 y 34 semanas de embarazo, sin infección clínica y sin trabajo de parto, tratadas aleatoriamente con antibiótico (clindamicina-gentamicina) o con placebo por 7 días, la $\mathrm{CH}$ aguda se encontró con más frecuencia en las pacientes con ICV (74\%) que en aquellas sin ICV (11\%) $(p<0,001)$, lo que sugiere que la $\mathrm{CH}$ es una infección intraamniótica, producto de una infección ascendente desde la vagina.

\section{Factores infecciosos causantes de resultados adversos obstétricos y neonatales en embarazadas con rotura prematura de membranas de pretérmino. Evidencia}

\section{INFECCIÓN CÉRVICO-VAGINAL/INVASIÓN MICROBIANA DE LA CAVIDAD AMNIÓTICA}

La presencia de ICV/IMCA se relaciona significativamente con infección materna, infección neonatal y duración del embarazo. En una serie ${ }^{5}$ de 80 embarazadas con RPM < 34 semanas, sin trabajo de parto, sin infección clínica, tratadas con clindamicina-gentamicina, la infección materna (corioamnionitis clínica, endometritis puerperal) se presentó más frecuentemente en los casos con IMCA e ICV (28\%) que en aquellos con ICV y $\sin$ IMCA (16\%) y que en aquellos sin IMCA y sin ICV $(0 \%)(p<0,05)$. La infección neonatal (sepsis, neumonía, enterocolitis necrotizante [EN]) ocurrió con más frecuencia en los casos con IMCA e ICV (20\%) que en aquellos con ICV y sin IMCA $(5 \%)$ y que en aquellos sin infección $(0 \%)(p<0,05)$. No tener ICV significa ausencia de riesgo de IMCA y de infección materna/neonatal. La latencia desde la admisión al parto en las mujeres con IMCA e ICV fue significativamente más breve $(5,4$ días) que en aquellas con ICV y sin IMCA (14,1 días) y que en aquellas sin infección (14,8 días) $(p<0,0001)$. Las bacterias aisladas en la ICV son similares a las encontradas en la IMCA, lo que apoya el concepto de que la infección intraamniótica es resultante del ascenso de microorganismos desde la vagina.

\section{Virulencia bacteriana}

Los resultados adversos obstétricos/neonatales son más frecuentes e intensos con algunos microorganismos que poseen mayor virulencia ${ }^{6}$. En un estudio retrospectivo, 96 pacientes con RPM de PT entre 24 y 34 semanas de embarazo, sin infección clínica y sin trabajo 
de parto, se manejaron con clindamicinagentamicina. Las pacientes con IMCA por SGB, F. nucleatum o $\mathrm{H}$. influenzae como único microorganismo aislado tuvieron mayor frecuencia y gravedad de resultados adversos obstétricos y neonatales que aquellas con IMCA por $U$. urealyticum como bacteria única aislada y que aquellas sin IMCA y sin ICV ${ }^{6}$. Resultados obstétricos adversos, corioamnionitis clínica ( $46 \%$ vs. $0 \%$ vs. $0 \% ; p<0,001)$, latencia al parto (2,8 días vs. 10,1 días vs. 18 días; $p<$ $0,0001)$, parto dentro de las 48 horas del ingreso $(64 \%$ vs. $21 \%$ vs. $3 \% ; p<0,0001)$ y parto dentro de los 7 días ( $91 \%$ vs. $53 \%$ vs. $14 \%$; $p<0,0001$ ), respectivamente. Resultados neonatales graves, infección (sepsis, neumonía o EN) $(36 \%$ vs. $5 \%$ vs. $0 \% ; p<0,01)$, asfixia severa y Apgar de 3 puntos o menos a los 5 minutos $(36 \%$ vs. $0 \%$ vs. $0 \% ; p<0,01)$ y muerte neonatal $(46 \%$ vs. $5 \%$ vs. $0 \% ; p<0,01)$, respectivamente ${ }^{6}$.

Los SGB son bacterias grampositivas muy frecuentes. Forman parte de la microbiota intestinal y colonizan a menudo el aparato genital inferior de la mujer. Se asocian con sepsis neonatal, neumonía congénita-connatal, muerte perinatal y secuelas neurológi$\mathrm{cas}^{14}$. Son las bacterias más frecuentes asociadas con morbilidad y mortalidad perinatal ${ }^{2,3}$.

F. nucleatum es una bacteria anaerobia gramnegativa que habita la cavidad oral y se asocia con enfermedad periodontal ${ }^{15}$. El feto-neonato se infecta habitualmente por vía hematógena transplacentaria, pero también existe evidencia de que puede ser por infección bacteriana ascendente a partir de microflora oral de la pareja.

$H$. influenzae es un bacilo gramnegativo pequeño. $H$. influenzae biotipo II es causa de morbilidad y mortalidad neonatal en pacientes con RPM de PT sin corioamnionitis clínica ${ }^{16,17}$. La infección intrauterina es secundaria a una infección ascendente desde la vagina, por microflora oral de la pareja ${ }^{5}$.

Ureaplasma spp. se ha asociado con PP y con enfermedad pulmonar crónica del infante ${ }^{18}$. Sin embargo, cuando está presente en la cavidad amniótica como única bacteria etiológica es menos frecuente su asociación con otros resultados adversos.

Otras bacterias causantes de morbilidad y mortalidad neonatal aisladas de la cavidad amniótica de pacientes con RPM son Escherichia coli ${ }^{19}$ y Morganella morganii ${ }^{20}$. Las placentas mostraron presencia de $\mathrm{CH}$ y de funisitis.

\section{CORIOAMNIONITIS HISTOLÓGICA/FUNISITIS}

La $\mathrm{CH}$ es la lesión más frecuente en la RPM y se relaciona con infección materna y neonatal. La funisitis es un marcador de morbilidad y de muerte neonatal por infección. En una serie ${ }^{7}$, la $\mathrm{CH}$ aguda en comparación con placentas sin $\mathrm{CH}$ se relacionó con infección materna (corioamnionitis clínica, endometritis puerperal) $(32 \%$ vs. $3 \% ; p<0,01)$ y con infección neonatal (neumonía, sepsis, EN) (27\% vs. 0\%; p < 0,01). La funisitis aguda en comparación con placentas sin funisitis se relacionó con muerte neonatal (29\% vs. $7 \%$; $p$ $<0,05)$, asfixia grave (33\% vs. $9 \%$; $p<0,01)$ e infección neonatal $(29 \%$ vs. $7 \%$; $p<0,05)$.

\section{SíndRome dE RESPUESTA FETAL INFLAMATORIA}

El síndrome de respuesta fetal inflamatoria (SRFI) es la elevación de la interleucina 6 (IL-6) en el plasma fetal $>11 \mathrm{pg} / \mathrm{ml}$ como consecuencia mayoritariamente de IMCA en pacientes con PP con o sin RPM. Es un factor de riesgo independiente para el desarrollo de morbilidad neonatal grave, síndrome de dificultad respiratoria (SDR), sepsis sospechada o comprobada, neumonía, displasia broncopulmonar, hemorragia intraventricular (HIV), leucomalacia periventricular o EN ${ }^{8}$. En una serie de 105 pacientes con PP y 52 pacientes con RPM, la prevalencia de morbilidad neonatal grave fue del $53,4 \%$. Los recién nacidos con morbilidad neonatal grave tuvieron concentraciones más altas de IL-6 en plasma que aquellos sin morbilidad neonatal grave (mediana: 14.0 vs. $5.2 \mathrm{pg} / \mathrm{ml} ; \mathrm{p}<0,005$ ). Se estableció un valor de corte de IL-6 en plasma fetal de $11 \mathrm{pg} / \mathrm{ml}$ para definir la presencia de una respuesta inflamatoria sistémica. La prevalencia de IL-6 en plasma fetal > $11 \mathrm{pg} / \mathrm{ml}$ fue del $49,3 \%$. Los fetos con concentraciones de IL-6 en plasma fetal $>11 \mathrm{pg} / \mathrm{ml}$ tuvieron una tasa más alta de morbilidad neonatal grave que aquellos con IL-6 $\leq 11 \mathrm{pg} / \mathrm{ml}(77.8 \% \text { vs. } 29.7 \% ; p<0,001)^{8}$. El análisis de regresión logística demostró que la concentración de IL-6 en plasma fetal fue un predictor independiente de morbilidad neonatal grave (odds ratio [OR]: 4.3; intervalo de confianza del 95\% [IC95\%]: 1-18.5), causa del PP (trabajo de parto prematuro o RPM), corioamnionitis clínica e intervalo desde la cordocentesis al parto.

En el estudio anteriormente mencionado ${ }^{6}$ de mujeres con RPT de PT se señaló que la IMCA por SGB, $F$. nucleatum $\circ \mathrm{H}$. influenzae, no obstante el uso de antibióticos, se relacionó con más frecuencia y mayor intensidad de resultados adversos neonatales, y con más frecuencia de $\mathrm{CH} /$ funisitis aguda. La IMCA produce en el feto un aumento de citocinas que conduce a una lesión orgánica multisistémica asociada con rápida morbimortalidad neonatal y con daños neurológicos 
posteriores (leucomalacia periventricular, HIV, parálisis cerebral), enfermedad crónica pulmonar y EN ${ }^{8,21-24}$. Los dramáticos resultados adversos neonatales encontrados permiten plantear la hipótesis de que el mayor daño en estos casos, además de por la mayor virulencia, podría producirse por un incremento de la respuesta fetal inflamatoria a estos microorganismos. Esta afirmación se sostiene por los siguientes hallazgos:

- La corioamnionitis clínica se presentó en la mitad de los casos de IMCA por SGB, F. nucleatum o Haemophilus influenzae. Se ha demostrado que la concentración de II-6 se eleva en la vena umbilical de recién nacidos cuyas madres tienen corioamnionitis clínica ${ }^{24}$.

- La CH apareció en todas las placentas y la funisitis se presentó en el $64 \%$ de las embarazadas con estas bacterias virulentas. Se ha demostrado que la funisitis es una manifestación histológica del SRF1 ${ }^{25}$.

- Las pacientes con IMCA por SGB, F. nucleatum o $\mathrm{H}$. influenzae presentaron latencia más corta hasta el parto y parto más frecuente dentro de las 48 horas del ingreso y dentro de los 7 días. Se sabe que el aumento de la respuesta fetal sistémica de citocinas proinflamatorias en las pacientes con IMCA y RPM de PT es seguido por el desencadenamiento inmediato del PP26.

En relación con la evidencia presentada, los resultados adversos obstétricos y especialmente los neonatales en pacientes con RPM de PT dependen de la existencia de IMCA o de ICV (o ambas), de la virulencia de los microorganismos aislados, del compromiso inflamatorio/infeccioso de la placenta $(\mathrm{CH}$, funisitis) y de la respuesta inflamatoria fetal; mecanismos que son interdependientes. La terapia antimicrobiana elegida deberá ser eficiente para tratar estos factores.

\section{Evaluación de los efectos de la}

administración de antibióticos en mujeres con rotura prematura de membranas de pretermino antes de las 37 semanas, en morbilidad infecciosa materna, morbilidad y mortalidad neonatal y desarrollo infantil a largo plazo

En esta revisión se incluyeron revisiones sistemáticas, metaanálisis, estudios seleccionados controlados aleatorizados que compararon antibióticos profilácticos o regímenes de antibióticos, entre sí o con placebo, o sin tratamiento, en mujeres con RPM de PT, y recomendaciones de sociedades científicas sobre el uso de antibióticos en la RPM de PT.

\section{Revisión sistemática Cochrane ${ }^{1}$}

Es la revisión más importante por el número de ensayos (22) y la población incluida (6872 mujeres y niños). Se prescribieron los siguientes esquemas de antibióticos:

- Ampicilina como antibiótico único por vía intravenosa (i.v.) y/o vía oral (v.o.) usada en esquema de días variables, en seis estudios ${ }^{27-32}$.

- Ampicilina asociada con diversos antibióticos: gentamicina, clindamicina, amoxicilina-ácido clavulánico; mezlocilina, ampicilina; amoxicilina, eritromicina; pivampicilina, metronidazol, eritromicina, administrados por vía i.v. y/o v.o., en cuatro estudios ${ }^{33-36}$.

- Eritromicina v.o. como antibiótico único en tres estudios $^{37-39}$.

- Amoxicilina-ácido clavulánico por vía i.v. en dos estudios ${ }^{40,41}$.

- Amoxicilina-ácido clavulánico más eritromicina v.o. en un estudio (ORACLE I) ${ }^{42}$.

- Ampicilina-sulbactam en un estudio ${ }^{43}$.

- Clindamicina asociada con gentamicina en un estudio ${ }^{11}$.

- Penicilina o derivados de la penicilina por vía i.v. en cuatro estudios ${ }^{44-47}$.

El uso de antibióticos después de la RPM se asoció con una reducción significativa de corioamnionitis (riesgo relativo [RR]: 0,66; IC95\%: 0,46-0,96), número de recién nacidos dentro de las 48 horas (RR: 0,71; IC95\%: 0,58-0,87) y dentro de los 7 días (RR: 0,79; IC95\%: 0,71-0,89), infección neonatal (RR: 0,67; IC95\%: 0,52-0,85), uso de surfactante (RR: 0,83; IC95\%: 0,720,96 ), oxigenoterapia (RR: 0,88; IC95\%: 0,81-0,96) y ecografía cerebral anormal antes del alta hospitalaria (RR: 0,81; IC95\%: 0,68-0,98). La administración de amoxicilina-ácido clavulánico se asoció con un mayor riesgo de EN neonatal (RR: 4,72; IC95\%: $1,57-14,23)^{41-43}$.

Se concluye que la indicación sistemática de antibióticos en mujeres con RPM de PT, entre 20 y 37 semanas de gestación, sin trabajo de parto y sin corioamnionitis clínica, se asocia con prolongación del embarazo y mejorías en una serie de morbilidades neonatales a corto plazo, pero sin reducción significativa en la mortalidad perinatal. No hay beneficio a largo plazo en la infancia. El antibiótico de elección no está claro, pero se debe evitar la amoxicilina-ácido clavulánico debido al mayor riesgo de EN neonatal. 


\section{Comentarios y reparos}

De los 22 trabajos, tres aportaron el $82 \%$ de las pacientes incluidas en esta revisión ${ }^{35,39,42}$. El más importante es el ORACLE I ${ }^{42}$, un ensayo multicéntrico con 4826 mujeres que recibieron aleatoriamente: el grupo 1, eritromicina $(250 \mathrm{mg})$; el grupo 2, amoxicilina-ácido clavulánico (325 mg); el grupo 3, ambas; y el grupo 4, placebo. En el grupo 1 se encontraron la mayoría de los beneficios mencionados anteriormente. Los recién nacidos de los grupos 2 y 3 no tuvieron ningún beneficio en comparación con placebo en cuanto a estos resultados. El grupo 2 tuvo una tasa significativamente mayor de EN neonatal.

Mercer et al. ${ }^{35,39}$, en dos estudios incluidos en esta revisión, usaron eritromicina v.o. hasta el parto y ampicilina más eritromicina por vía i.v. durante 2 días, y luego amoxicilina más eritromicina v.o. durante 5 días. Cabe mencionar que estos esquemas no son eficaces para tratar anaerobios ni enterobacterias. En más de la mitad de los trabajos (13) se usó un solo antibiótico, ampicilina o eritromicina o penicilina o derivados de la penicilina; estos esquemas (10) no son eficaces en el tratamiento de anaerobios, enterobacterias ni micoplasmas. Cuatro de los 22 trabajos estudiaron la microbiota urogenital. Dos pesquisaron SGB vaginoperineal ${ }^{6,7}$, uno realizó estudio microbiológico urogenital ${ }^{14} \mathrm{y}$ otro investigó la microbiota de la cavidad amniótica y del tracto genital inferior ${ }^{36}$. El estudio microbiológico de la cavidad amniótica y de la microbiota vaginal es importante para el uso apropiado de los antimicrobianos ${ }^{5,6,9}$.

\section{REVISIÓN SISTEMÁTICA}

Kenyon et al. ${ }^{4}$ incluyeron en su revisión 14 ensayos ${ }^{11,29,34-40,42,44,46-48}$ con 6559 mujeres. Los antibióticos se asociaron con una reducción significativa de la infección materna y de la corioamnionitis. Además, redujeron el número de recién nacidos dentro de las 48 horas y dentro de los 7 días, y disminuyeron las siguientes morbilidades: infección neonatal (RR: 0,67; IC95\%: 0,52-0,85), hemocultivo positivo (RR: 0,75; IC95\%: 0,600,93), uso de surfactante (RR: 0,83; IC95\%: 0,72-0,96), oxigenoterapia (RR: 0,88; IC95\%: 0,81-0,96) y ecografía cerebral anormal antes del alta hospitalaria (RR: 0,82; IC95\%: 0,68-0,99). La mortalidad perinatal no se redujo. La amoxicilina-ácido clavulánico se asoció con un aumento significativo en el riesgo de EN (RR: 4,60; IC95\%: 1,98-10,72). Se concluye que la administración de antibióticos en la RPM de PT es beneficiosa tanto para las mujeres como para los recién nacidos. Estos datos apoyan el uso sistemático de antibióticos en estos casos. El beneficio se obtiene con las penicilinas y la eritromicina, pero la eritromicina tiene resultados más sólidos por su uso en un mayor número de casos. La amoxicilina-ácido clavulánico no debe usarse por el riesgo de EN neonatal.

\section{MetaAnálisis de ensayos controlados ALEATORIZADOS}

El metaanálisis de Chatzakis et al..$^{49}$ incluyó 20 estu$\operatorname{dios}^{11,27,29-31,33-35,38-42,44-47,50-52}$, con un total de 7169 participantes y 15 regímenes terapéuticos. Se compararon antibióticos profilácticos, o regímenes de antibióticos, entre sí o con placebo, en mujeres con RPM de PT. Los resultados primarios fueron la mortalidad neonatal y la corioamnionitis. Los resultados secundarios incluyeron otras medidas de morbilidad perinatal. Se evaluó la calidad de la evidencia mediante las guías GRADE (Grading of Recommendations, Assessment, Development and Evaluation). Para el resultado de corioamnionitis, el antibiótico fue superior a placebo y a sin tratamiento; el esquema más eficiente fue clindamicina + gentamicina (RR: 0,19 IC95\%: 0,05-0,83), seguido de penicilina (RR: 0,31, IC95\%: 0,16-0,60), ampicilina-sulbactam + amoxicilina-ácido clavulánico (RR: 0,32, IC95\%: 0,12-0,92), ampicilina (RR: 0,52, IC95\%: 0,34-0,81) y eritromicina + ampicilina + amoxicilina (RR: 0,71; IC95\%: 0,55-0,92). Para reducir la sepsis neonatal, la eritromicina fue el único fármaco efectivo (RR: 0,74; IC95\%: 0,56-0,97). Para disminuir el SDR fueron eficaces clindamicina + gentamicina (RR: 0,32; IC95\%: 0,11-0,89) y eritromicina + ampicilina + amoxicilina (RR: 0,83; IC95\%: 0,69-0,99). Para reducir las tasas de HIV fueron efectivas ampicilina (RR: 0,42; IC95\%: 0,20-0,92) y penicilina (RR: 0,49; IC95\%: 0,25-0,96). Ninguno de los antibióticos fue significativamente más efectivo que el placebo para reducir las tasas de muerte neonatal, muerte perinatal y EN. Se concluye que varios antibióticos son más efectivos que el placebo para disminuir la tasa de corioamnionitis en la RPM de PT, pero ninguno de ellos es consistentemente superior. La calidad general de la evidencia es baja y debe actualizarse, porque puede haber aparecido resistencia microbiana a algunos antibióticos, mientras que otros están infrarrepresentados en la evidencia existente.

\section{Comentarios}

La combinación de clindamicina + gentamicina fue más eficaz para tratar la corioamnionitis y el SDR ${ }^{11}$, la 
Tabla 1. Sociedades y Organizaciones Médicas de Obstetricia y Ginecología. Recomendaciones sobre uso de antibióticos en pacientes con RPM de PT

\begin{tabular}{|c|c|c|}
\hline $\begin{array}{l}\text { Sociedades y Organizaciones } \\
\text { Médicas }\end{array}$ & $\begin{array}{l}\text { Dosis y uso } \\
\text { Inicio }\end{array}$ & $\begin{array}{l}\text { Dosis y uso } \\
\text { Seguido de }\end{array}$ \\
\hline $\begin{array}{l}\text { American College of Obstetricians } \\
\text { and Gyneco Gynecologists (ACOG) }\end{array}$ & $\begin{array}{l}\text { ampicilina } 2 \mathrm{~g} \text { IV c/6 h + eritromicina } 250 \mathrm{mg} \mathrm{IV} \mathrm{c/6} \\
\mathrm{h} \text { por } 2 \text { días }\end{array}$ & $\begin{array}{l}\text { amoxicilina } 250 \mathrm{mg} 0 \mathrm{c} / 8 \mathrm{~h}+ \\
\text { eritromicina } 333 \mathrm{mg} 0 \mathrm{c} / 8 \mathrm{~h} \text { por } 5 \text { días }\end{array}$ \\
\hline $\begin{array}{l}\text { Sociedad Española de Ginecología y } \\
\text { Obstetricia (SEGO) })^{54}\end{array}$ & $\begin{array}{l}\text { ampicilina } 2 \mathrm{~g} \text { IV c/6 h + eritromicina } 250 \mathrm{mg} \mathrm{IV} \mathrm{c/6} \\
\mathrm{h} \text { por } 2 \text { días }\end{array}$ & amoxicilina + eritromicina 0 por 5 días \\
\hline $\begin{array}{l}\text { Royal College of Obstetricians and } \\
\text { Gynaecologists (RCOG) }{ }^{55}\end{array}$ & eritromicina 0 por 10 días & - \\
\hline $\begin{array}{l}\text { National Institute for Health and } \\
\text { Care Excellence (NICE) }{ }^{56}\end{array}$ & $\begin{array}{l}\text { eritromicina } 250 \mathrm{mg} 0 \text { c/6 h máximo por } 10 \text { días } 0 \\
\text { hasta el parto. Con intolerancia a la eritromicina } \\
\text { usar penicilina oral }\end{array}$ & - \\
\hline World Health Organization $(\mathrm{WHO})^{57}$ & $\begin{array}{l}\text { eritromicina } 250 \mathrm{mg} 0 \mathrm{c} / 6 \mathrm{~h} \text { por } 10 \text { días. Con } \\
\text { intolerancia a la eritromicina usar amoxicilina oral }\end{array}$ & - \\
\hline $\begin{array}{l}\text { Society of Obstetricians and } \\
\text { Gynaecologists of Canada (SOGC) })^{58}\end{array}$ & $\begin{array}{l}\text { ampicilina } 2 \mathrm{~g} \mathrm{IV} \mathrm{c/6} \mathrm{h} \mathrm{+} \mathrm{eritromicina} 250 \mathrm{mg} \mathrm{IV} \mathrm{c/6} \\
\mathrm{h} \text { x } 48 \mathrm{~h} 0 \text { eritromicina } 250 \mathrm{mg} 0 \mathrm{c} / 6 \mathrm{~h} \text { por } 10 \text { días }\end{array}$ & $\begin{array}{l}\text { amoxicilina } 250 \mathrm{mg} 0 \mathrm{c} / 8 \mathrm{~h}+ \\
\text { eritromicina } 333 \mathrm{mg} 0 \mathrm{c} / 8 \mathrm{~h} \text { por } 5 \text { días }\end{array}$ \\
\hline MINSAL, Guía Perinatal ${ }^{59}$ & $\begin{array}{l}\text { clindamicina } 600 \mathrm{mg} \text { IV c/8 h por } 7 \text { días + } \\
\text { gentamicina } 240 \mathrm{mg} / \text { día IV por } 7 \text { días + eritromicina } \\
500 \mathrm{mg} \text { c/6 h } 0 \text { por } 7 \text { días }\end{array}$ & - \\
\hline
\end{tabular}

IV: intravenosa; IM: intramuscular; 0: oral; h: horas.

eritromicina para tratar la sepsis neonatal ${ }^{34-36}$ y la ampicilina para tratar la HIV28,30-32. La eficacia de la combinación clindamicina + gentamicina es teóricamente posible por los espectros antimicrobianos complementarios de las dos y sus altas concentraciones en la placenta y las membranas. Sin embargo, ninguno de estos antibióticos es efectivo contra Ureaplasma spp.

\section{Sociedades y organizaciones médicas de obstetricia y ginecología}

\section{Recomendaciones sobre uso de antibióticos en pacientes con RPM de PT propuestas por Sociedades y Organizaciones médicas de Obstetricia y Ginecología}

Se seleccionaron las principales sociedades y de organizaciones médicas de obstetricia y ginecología de países europeos y americanos

\section{Manuscritos Seleccionados}

\section{Antibióticos y resultados obstétricos y neonatales}

Ovalle et al. ${ }^{11}$ realizaron un estudio prospectivo, aleatorizado antibiótico-placebo, con 88 mujeres con RPM entre 22 y 34 semanas, sin corioamnionitis clínica y sin trabajo de parto. De estas, 42 recibieron clindamicina (600 mg c/6 h i.v.) + gentamicina (4 mg/kg/día i.v.) por 2 días, y luego clindamicina (300 mg c/6 h v.o.) + gentamicina (2 mg/kg/día c/12 h intramuscular) por 5 días, y 46 recibieron placebo. La IMCA se evaluó mediante cultivo positivo de líquido amniótico. La ICV se evaluó por la presencia de vaginosis bacteriana o vaginitis aeróbica 0 micoplasmas genitales, junto con aumento del recuento de glóbulos blancos > 10/campo $400 \times$. Con antibióticos, en comparación con placebo, se prolongó el embarazo (latencia desde ingreso al parto, mediana: 10,5 vs. 4 días; $p<0,05)$, de manera más marcada en pacientes con ICV y sin IMCA (mediana: 20 vs. 7 días; $p<0,05$ ), y se redujo la morbilidad infecciosa materna (endometritis puerperal, corioamnionitis clínica; $4.8 \%$ vs. $28.9 \%$; $p<0,01$ ), efecto más marcado en el grupo con IMCA (corioamnionitis clínica; $9.1 \%$ vs. $45.5 \% ; p<0,01$ ). En el grupo de madres con antibióticos, los neonatos tuvieron mayor peso al nacer ( 1849 vs $1645 \mathrm{~g} ; \mathrm{p}=0,05)$ y redujeron el SDR $(9.5 \%$ vs. $30,2 \%$; $p<0,05)$ y la admisión a la unidad de cuidado intensivo neonatal ( $54.8 \%$ vs. $86.0 \%$; $p<0,01)$.

\section{Comentarios}

El efecto de los antibióticos, prolongar la gestación, más marcado en mujeres con ICV y sin IMCA, es el 
fundamento para proponer que en las pacientes con RPM de PT se utilice la terapia antimicrobiana en todos los casos con ICV. En ausencia de ICV y de IMCA, las pacientes no se benefician con el uso de antibióticos y estos deben suspenderse. La reducción del SDR con el uso de antibióticos (no se administraron corticoides) puede explicarse por la prolongación significativa del embarazo. En este estudio no disminuyó la infección neonatal, por un probable sesgo en el manejo. Se administraron antibióticos a los recién nacidos con peso $<2000 \mathrm{~g}$ por norma y cuando los cultivos de la madre fueron positivos en el grupo de placebo. Este manejo en la unidad de neonatología pudo disminuir la tasa de infección neonatal.

\section{ORACLE I, evaluación a 7 años ${ }^{60}$}

A la edad de 7 años se evaluaron los niños nacidos de las 4148 mujeres que habían completado el ensayo ORACLE I y que eran elegibles para el seguimiento, con un cuestionario estructurado aplicado a los padres para evaluar el estado de salud del niño. El deterioro funcional se definió como la presencia de cualquier grado de deterioro funcional (grave, moderado o leve) según el Mark III Multi-Attribute Health Status. Los resultados educativos se evaluaron con los resultados de las pruebas curriculares nacionales para niños residentes en Inglaterra. El resultado se determinó para 3298 (75\%) niños elegibles. No hubo diferencias en la proporción de niños con algún deterioro funcional después de la prescripción de eritromicina, con o sin amoxicilina-ácido clavulánico, en comparación con los nacidos de madres que no recibieron eritromicina (38.3\% vs. 40,4\%; OR: 0,91; IC95\%: 0,79-1.05); o después de la prescripción de amoxicilina-ácido clavulánico, con o sin eritromicina, en comparación con los nacidos de madres que no recibieron amoxicilina-ácido clavulánico (40,6\% vs. 38,1\%; OR: 1,11; IC 95\%: 0,961.28). Ninguno de los antibióticos usados tuvo un efecto significativo en el nivel general de dificultades conductuales experimentadas ni en las proporciones de niños que alcanzaron cada nivel en lectura, escritura o matemáticas en la etapa clave uno. Se concluye que la prescripción de antibióticos para mujeres con RPM de PT parece tener poco efecto sobre la salud de los niños a los 7 años de edad ${ }^{53-60}$.

\section{Comentarios}

La explicación de la ausencia de beneficios a largo plazo con los antibióticos en pacientes con RPM de PT en este estudio no es clara. Una interpretación podría relacionarse con la breve exposición a los antibióticos de las pacientes con RPM e IMCA, ya que la mayoría de ellas $(60 \%)$ tuvieron el parto dentro de la primera semana de la admisión; otra, que la asociación de antimicrobianos usados fuera insuficiente para tratar los diferentes microorganismos presentes. En ambas condiciones es muy posible que no se erradicara la infección intraamniótica. Es trascendental que las mujeres con evidencia de infección clínica sean tratadas con antibióticos, ya que la corioamnionitis clínica sigue siendo una causa importante de muerte materna, fetal y neonatal.

\section{Antibióticos en la RPM de PT y resultados placentarios}

Ovalle et al. ${ }^{12}$ realizaron un estudio prospectivo, aleatorizado antibiótico-placebo, con 71 mujeres con RPM, embarazo < 34 semanas, sin corioamnionitis clínica y sin trabajo de parto. Se usó clindamicina-gentamicina (dosis, vía y duración de tratamiento mencionados en el trabajo anterior ${ }^{11}$ ) y las placentas se enviaron a estudio histológico. El objetivo fue determinar si la administración de antibióticos se asocia con una reducción en la tasa de $\mathrm{CH}$ y de funisitis. $\mathrm{La} \mathrm{CH}$ se evaluó por la presencia de leucocitos polimorfonucleares en las membranas fetales o en la placa subcoriónica. La funisitis se evaluó por la inflamación aguda del cordón umbilical representada por la existencia de leucocitos polimorfonucleares en la pared del vaso umbilical. Con antibióticos se redujo la $\mathrm{CH}(46 \%$ vs. $69 \%$; $p<0,05)$, efecto más intenso en las mujeres con IMCA o ICV (58\% vs. $89 \% ; p<0,01)$ y se incrementó la frecuencia de placenta sin lesiones ( $29 \%$ vs. $6 \% ; p<0,01)$. Este estudio proporciona una mejor comprensión de la etiología de la infección intrauterina ascendente, al establecer que la $\mathrm{CH}$ se reduce con el uso de antibióticos. Por otro lado, la funisitis representa una condición fetal grave con una pobre respuesta a los antimicrobianos. Estos resultados requieren esfuerzos renovados para identificar las primeras etapas de infección del tracto genital inferior en las pacientes en riesgo.

\section{Nuevo esquema antimicrobiano}

Lee et al. ${ }^{61}$ realizaron un estudio retrospectivo que comparó los resultados perinatales en 314 pacientes con RPM < 34 semanas que recibieron ampicilina y/o cefalosporinas $(n=195)$ entre 1993 y 2003, frente a ceftriaxona + claritromicina y metronidazol $(n=119)$ 
Tabla 2. Esquemas de antibióticos recomendados de acuerdo con la evidencia presentada

\begin{tabular}{|c|c|c|}
\hline Esquemas de antibióticos & $\begin{array}{l}\text { Inicio } \\
\text { Dosis y uso }\end{array}$ & $\begin{array}{l}\text { Seguido de } \\
\text { Dosis y uso }\end{array}$ \\
\hline $\begin{array}{l}\text { Ceftriaxona + claritromicina + } \\
\text { metronidazol }\end{array}$ & $\begin{array}{l}\text { Ceftriaxona } 1 \mathrm{~g} \text { i.v. por } \\
\text { día+claritromicina } 500 \mathrm{mg} \mathrm{c} / 12 \mathrm{~h} \text { v.o. } \\
\text { + metronidazol } 500 \mathrm{mg} \mathrm{c} / 8 \mathrm{~h} \text { i.v. } \\
\text { administrados hasta el parto }\end{array}$ & $\begin{array}{l}\text { Metronidazol v.o. hasta } 4 \text { semanas, si el parto no } \\
\text { ocurrió }\end{array}$ \\
\hline $\begin{array}{l}\text { Clindamicina + gentamicina + } \\
\text { eritromicina o azitromicina }\end{array}$ & $\begin{array}{l}\text { Clindamicina } 600 \mathrm{mg} \mathrm{c} / 6 \text { h i.v. }+ \\
\text { gentamicina } 4 \mathrm{mg} / \mathrm{kg} / \text { día i.v. por } 2 \\
\text { días }\end{array}$ & $\begin{array}{l}\text { Clindamicina } 300 \mathrm{mg} \mathrm{c} / 6 \text { h v.o. por } 5 \text { días + } \\
\text { gentamicina } 2 \mathrm{mg} / \mathrm{kg} / \text { día i.v. } 0 \text { i.m. por } 5 \text { días + } \\
\text { eritromicina } 500 \mathrm{mg} \mathrm{c} / 6 \mathrm{~h} \text { v.o. hasta el parto } 0 \\
\text { azitromicina } 500 \mathrm{mg} / \text { día v.o. hasta el parto }\end{array}$ \\
\hline $\begin{array}{l}\text { Clindamicina + cefazolina o cefuroxima } \\
\text { + eritromicina }\end{array}$ & $\begin{array}{l}\text { Clindamicina } 600 \mathrm{mg} \mathrm{c} / 6 \mathrm{~h} \text { i.v. } \\
\text { + cefazolina } 1 \mathrm{~g} \mathrm{c} / 6 \mathrm{~h} \text { i.v. } 0 \\
\text { cefuroxima } 750 \mathrm{mg} \mathrm{c} / 8 \mathrm{~h} \text { i.v., ambos } \\
\text { por } 48 \mathrm{~h}\end{array}$ & $\begin{array}{l}\text { Clindamicina } 300 \mathrm{mg} \mathrm{c} / 6 \mathrm{~h} \text { v.o. + cefradina } 500 \mathrm{mg} \\
\mathrm{c} / 6 \mathrm{~h} \text { v.0. } 0 \text { cefuroxima } 250 \mathrm{mg} \mathrm{c} / 12 \mathrm{~h} \text { v.0., ambos } \\
\text { hasta el parto }\end{array}$ \\
\hline
\end{tabular}

entre 2003 y 2012. La infección/inflamación intraamniótica se evaluó mediante cultivo positivo de líquido amniótico o una concentración elevada de metaloproteinasa de matriz 8 (423 ng/ml) en el líquido amniótico. El segundo régimen antimicrobiano incluyó ceftriaxona (1 g c/24 h i.v.), claritromicina (500 mg c/12 h v.o.) y metronidazol (500 mg c/8 h i.v.) administrados hasta el parto, excepto el metronidazol, que se administró como máximo 4 semanas.

Las pacientes tratadas con ceftriaxona + claritromicina y metronidazol, en comparación con las que recibieron ampicilina o cefalosporinas, tuvieron un intervalo entre admisión y parto más prologado (mediana: 23 vs. 12 días; $p<0,01$ ), tasas más bajas de $\mathrm{CH}$ aguda (50,5\% vs. $66,7 \%$; $p<0,05)$ y de funisitis $(13,9 \%$ vs. $42,9 \% ; p<0,001)$, tasas más bajas de HIV $(2,1 \%$ vs. $19,0 \% ; p<0,001)$ y de parálisis cerebral $(0 \%$ vs. $5,7 \%$; $p<0,05)$, y mejor resultado perinatal en embarazos con infección/inflamación intraamniótica, pero no en aquellos sin infección/inflamación intraamniótica, en el análisis de subgrupos. Se concluye que la combinación de ceftriaxona, claritromicina y metronidazol mejora el resultado perinatal en pacientes con RPM de $\mathrm{PT}$, prolonga el período de latencia, reduce la $\mathrm{CH}$ y la funisitis aguda, y mejora los resultados neonatales.

\section{Comentario}

Esta es la mejor asociación publicada, por su eficacia en el tratamiento de micoplasmas genitales, microorganismos presentes en la vaginosis bacteriana y la vaginitis aeróbica, y bacterias de mayor virulencia, y por sus mejores resultados maternos neonatales y prolongación del periodo de latencia. Es una asociación de antimicrobianos segura. Cabe destacar la reducción de la corioamnionitis y de la funisitis histológica aguda con este esquema, a diferencia de clindamicina-gentamicina, que logró disminuir la $\mathrm{CH}$ aguda, pero no la funisitis ${ }^{12}$.

\section{Eficacia de los esquemas de antibióticos según la evidencia analizada}

\section{La eficacia de los esquemas de antibióticos publicados es:}

- Para todos los resultados adversos: 1) clindamicina + gentamicina ${ }^{11,12}$, y luego 2) penicilina ${ }^{45-47}$ y 3) ampicilina, eritromicina + ampicilina + amoxicilina ${ }^{3,53,58}$.

- Para patología neonatal: sepsis, eritromicina ${ }^{37-39,55-57}$; SDR, 1) clindamicina + gentamicina ${ }^{11,12}$ y 2) eritromicina + ampicilina + amoxicilina ${ }^{3,53,58}$; HIV, ampicilina $^{27,32}$ y penicilinas ${ }^{45-47}$. La amoxicilina-ácido clavulánico no debe usarse por riesgo de EN neonatal.

- Para reducir la $\mathrm{CH}:$ 1) ceftriaxona + claritromicina + metronidazol ${ }^{61}$ y 2) clindamicina+ gentamicina ${ }^{11,12}$.

- Para reducir la funisitis: ceftriaxona + claritromicina + metronidazol $^{61}$.

\section{Ceftriaxona + Claritromicina + METRONIDAZOL ${ }^{61}$}

Es la mejor asociación, por su eficacia en el tratamiento de micoplasmas y microorganismos presentes en la vaginosis bacteriana y en la vaginitis aeróbica, y bacterias de mayor virulencia, y sus mejores resultados maternos neonatales, prolongación del periodo de latencia y reducción de la corioamnionitis y la funisitis histológica aguda. Es un esquema seguro y con una recomendación $\mathrm{A}^{64}$. 


\section{Clindamicina + Gentamicina ${ }^{11,12}$ - ERITOMICINA ${ }^{37-39,55-57}$ O AZITROMICINA 63}

Es la segunda mejor asociación, por su eficacia en el tratamiento de micoplasmas, microorganismos presentes en la vaginosis bacteriana y en la vaginitis aeróbica, y bacterias de mayor virulencia, buena seguridad y bajo costo. Proporciona buenos resultados maternos neonatales. Reduce la $\mathrm{CH}$, pero no la funisitis, y aumenta la frecuencia de placenta sin lesiones. Alcanza una buena concentración en el líquido amniótico y las membranas ovulares. Con eritromicina o azitromicina disminuye la sepsis neonatal ${ }^{37-39,55-57,63}$. Tiene un grado de recomendación $\mathrm{A}^{64}$.

\section{Clindamicina ${ }^{11,12}$ - Cefazolina ${ }^{51}$ o Cefuroxima ${ }^{61}$ - ERITROMICINA ${ }^{37-39,55-57}$ O AZITROMICINA 63}

Es la tercera mejor asociación, por su eficacia en el tratamiento de micoplasmas, microorganismos presentes en la vaginosis bacteriana y en la vaginitis aeróbica, $\mathrm{y}$ bacterias de mayor virulencia. Muestra seguridad y costos prudentes.

\section{Conclusiones}

La RPM de PT es causa de hasta un tercio de los PP. La IMCA y la ICV están presentes en el $50 \%$ y el $74 \%$, respectivamente, de estos casos. Los resultados adversos, infección materna, infección neonatal, duración del embarazo y lesiones inflamatorias placentarias $\mathrm{CH} /$ funisitis aguda, se presentan significativamente más con IMCA/ ICV y son más frecuentes e intensos en la IMCA por SGB, $F$. nucleatum y $H$. influenzae, microorganismos que poseen gran virulencia. El SRFI es un factor de riesgo independiente para el desarrollo de morbilidad neonatal grave y probablemente es de más gravedad con las bacterias de mayor virulencia y en los casos con funisitis.

La indicación sistemática de antibióticos prolonga el embarazo, disminuye la corioamnionitis clínica, la $\mathrm{CH}$ y la funisitis aguda, y reduce una serie de morbilidades neonatales a corto plazo, pero no disminuye la mortalidad perinatal ni las secuelas tardías. El esquema antimicrobiano de elección para reducir los resultados adversos obstétricos neonatales debe ser eficiente frente a la mayoría de los agentes etiológicos descritos en la IMCA y la ICV, y frente a los factores infecciosos implicados en la gravedad de la infección. Además, deben administrarse de manera oportuna e intensiva en las primeras 48 a 72 h por vía i.v., para luego continuar con presentaciones orales hasta el parto.

\section{Financiamiento}

El manuscrito no requirió financiamiento.

\section{Conflicto de intereses}

Los autores no tienen conflictos de intereses.

\section{Responsabilidades éticas}

Protección de personas y animales. Los autores declaran que para esta investigación no se han realizado experimentos en seres humanos ni en animales.

Confidencialidad de los datos. Los autores declaran que han seguido los protocolos de su centro de trabajo sobre la publicación de datos de pacientes.

Derecho a la privacidad y consentimiento informado. Los autores declaran que en este artículo si aparecen datos de pacientes.

\section{Bibliografía}

1. Kenyon S, Boulvain M, Neilson JP. Antibiotics for preterm rupture of membranes. Cochrane Database Syst Rev. 2013;(12):CD001058.

2. Ovalle A, Kakarieka E, Rencoret G, Fuentes A, del Río MJ, Morong C, et al. Risk factors for preterm deliveries in a public hospital. Rev Med Chile. 2012;140:19-29.

3. Ovalle A, Kakarieka E, Díaz M, García Huidobro T, Acuña J, Fuentes A, et al. Mortalidad perinatal en el parto prematuro entre 22 y 34 semanas en un hospital público de Santiago, Chile. Rev Chil Obstet Ginecol. 2012;77:263-70.

4. Kenyon S, Boulvain M, Neilson J. Antibiotics for preterm rupture of the membranes: a systematic review. Obstet Gynecol. 2004;104:1051-7.

5. Ovalle A, Martínez MA, Giglio MS, Poblete JP, Fuentes A, Gómez R, et al. Microbiología aislada en la rotura prematura de membranas de pretérmino. Relación con morbilidad infecciosa materna neonatal e intervalo rotura de membranas-parto. Rev Chil Obstet Ginecol. 1995;60:252-62.

6. Ovalle A, Gómez R, Martínez MA, Kakarieka E, Fuentes A, Aspillaga $C$, et al. Invasión microbiana de la cavidad amniotica en la rotura de membranas de pretérmino. Resultados maternoneonatales y patología placentaria según microorganismo aislado. Rev Med Chile. 2005;133:51-61.

7. Ovalle A, Martínez MA, Kakarieka E, Gómez R, Torres J, Fuentes A, et al. Histopatología de la placenta en la rotura prematura de membranas de pretérmino. Relación con la microbiología aislada y con los resultados materno neonatales. Rev Med Chil. 1998;126:930-42.

8. Gómez R, Romero R, Ghezzi F, Yoon BH, Mazor M, Berry SM. The fetal inflammatory response syndrome. Am J Obstet Gynecol. 1998;179:194-202.

9. Romero R, Miranda J, Chaemsaithong P, Chaiworapongsa T, Kusanovic JP, Dong Z, et al. Sterile and microbial-associated intra-amniotic inflammation in preterm prelabor rupture of membranes. J Matern Fetal Neonatal Med. 2015;28:1394-409.

10. DiGiulio DB, Romero R, Kusanovic JP, Gómez R, Kim CJ, Seok K, et al. Prevalence and diversity of microbes in the amniotic fluid, the fetal inflammatory response, and pregnancy outcome in women with preterm pre-labor rupture of membranes. Am J Reprod Immunol. 2010;64:38-57.

11. Ovalle A, Gómez R, Martínez MA, Rubio R, Fuentes A, Romero R, et al. Antibiotic therapy in patients with preterm premature rupture of membranes: a prospective, randomized, placebo controlled study with microbiologic assessment of the amniotic cavity and lower genital tract. Prenat Neonatal Med. 1997;2:213-22. 
Rev Chil Obstet Ginecol. 2021;86(5)

12. Ovalle A, Martínez MA, Kakarieka E, Gómez R, Rubio R, Valderrama O, et al. Antibiotic administration in patients with preterm premature rupture of membranes reduces the rate of histological chorioamnionitis: a prospective, randomized, controlled study. J Matern Fetal Neonatal Med. 2002;12:35-41.

13. Robertson JA, Vekris A, Bebear C, Stemke GW. Polymerase chain reaction using 16S rRNA gene sequences distinguishes the two biovars of Ureaplasma urealyticum. J Clin Microbiol. 1993;31:824-30.

14. Heath PT. Perinatal group B streptococcal disease. Best Pract Res Clin Obstet Gynaecol. 2007;21:411-24.

15. Vander Haar EL, So J, Gyamfi-Bannerman C, Han YW. Fusobacterium nucleatum and adverse pregnancy outcomes: epidemiological and mechanistic evidence. Anaerobe. 2018;50:55-9.

16. Ovalle A, Martínez MA, Torres J, Cona E, Poblete P. Parto prematuro con rotura de membranas y transmisión fetal de Haemophilus influenzae. Rev Chil Pediatr. 1994;65:158-60.

17. Martínez MA, Ovalle A, Ulloa MT, Vidal R. Role of Haemophilus influenzae in intra-amniotic infection in patients with preterm rupture of membranes. Eur J Clin Microb Infect Dis. 1999;18:890-3.

18. Kafetzis DA, Skevaki CL, Skouteri V, Gavrili S, Peppa K, Kostalos C, et al. Maternal genital colonization with Ureaplasma urealyticum promotes preterm delivery: association of the respiratory colonization of premature infants with chronic lung disease and increased mortality. Clin Infect Dis. 2004;39:1113-22.

19. Ovalle A, García M, Oda F, Alvarado S, Martínez MA. Meningitis neonatal precoz causada por transmisión vertical de Escherichia coli productora de beta-lactamasa de espectro extendido en parto prematuro con rotura prematura de membranas. Rev Chil Obst Ginec. 2017:82:621-5.

20. Ovalle A, Martínez MA, Kakarieka E, García Mirna, Salinas A. Fata neonatal sepsis caused by vertical transmission of Morganella morganii. Report of one case. Rev Med Chile. 2009;137:1201-4.

21. Bracci R, Buonocore G. Chorioamnionitis: a risk factor for fetal and neonatal morbidity. Biol Neonate. 2003;83:85-96.

22. Vigneswaran R. Infection and preterm birth: evidence of a common causal relationship with bronchopulmonary dysplasia and cerebral palsy. J Paediatr Child Health. 2000;36:293-6.

23. Schendel DE, Schuchat $A$, Thorsen $P$. Public health issues related to infection in pregnancy and cerebral palsy. Ment Retard Dev Disabil Res Rev. 2002:8:39-45.

24. Chaiworapongsa T, Romero R, Kim JC, Kim YM, Blackwell SC, Yoon BH et al. Evidence for fetal involvement in the pathologic process of clinical chorioamnionitis. Am J Obstet Gynecol. 2002;186:1178-82.

25. Pacora P, Chaiworapongsa T, Maymon E, Kim YM, Gomez R, Yoon BH et al. Funisitis and chorionic vasculitis: the histological counterpart of the fetal inflammatory response syndrome. J Matern Fetal Neonatal Med. 2002;11:18-25.

26. Romero R, Gomez R, Ghezzi F, Yoon BH, Mazor M, Edwin SS, et al A fetal systemic inflammatory response is followed by the spontaneous onset of preterm parturition. Am J Obstet Gynecol. 1998:179:186-93.

27. Amon E, Lewis SV, Sibai BM, Villar MA, Arheart KL. Ampicillin prophylaxis in preterm premature rupture of the membranes: a prospective randomized study. Am J Obstet Gynecol. 1988;159:539-43.

28. Camli L, Mavunagacioglu S, Bostanci A, Camli S, Soylu F. Antibiotherapy in preterm premature rupture of membrane. Does it affect the latent period and infectious morbidity? Jinekoloji Ve Obstetrik Dergisi. 1997;11:138-42.

29. Grable IA, García PM, Perry D, Socol ML. Group B streptococcus and preterm premature rupture of membranes: a randomized, double-blind clinical trial of antepartum ampicillin. Am J Obstet Gynecol. 1996;175:1036-42.

30. Morales WJ, Angel JL, O'Brien WF, Knuppel RA. Use of ampicillin and corticosteroids in premature rupture of membranes: a randomized study. Obstet Gynecol. 1989;73:721-6.

31. Owen J, Groome LJ, Hauth JC. Randomised trial of prophylactic therapy after preterm amnion rupture. Am J Obstet Gynecol. 1993;169:976-81.

32. Segel SY, Miles AM, Clothier B, Parry S, Macones GA. Duration of antibiotic therapy after preterm premature rupture of fetal membranes. Am J Obstet Gynecol. 2003:189:799-802.

33. Christmas JT, Cox SM, Andrew W, Dax J, Leveno KJ, Gilstrap LC. Expectant management of preterm ruptured membranes: effects of antimicrobial therapy. Obstet Gynecol. 1992:80:759-62.

34. Johnston MM, Sánchez-Ramos L, Vaughan AJ, Todd MW, Benrubi GI. Antibiotic therapy in preterm premature rupture of membranes: a randomized, prospective, double-blind trial. Am J Obstet Gynecol. 1990;163:743-7.

35. Mercer BM, Miodovnik M, Thurnau GR, Goldenberg RL, Das AF, Ramsey RD, et al. Antibiotic therapy for reduction of infant morbidity after preterm premature rupture of the membranes. JAMA. 1997:278:989-95.

36. Svare J. Preterm delivery and subclinical uro-genital infection [thesis] Denmark: Department of Obstetrics and Gynaecology Rigshospitalet University of Copenhagen; 1997.

37. García-Burguillo A, Hernández-García JM, de la Fuente P. Profilaxis con eritromicina en gestaciones pretermino con rotura prematura de las membranas amnioticas. Clin Invest Gin Obst. 1995;(233):96-100.
38. McGregor JA, French JI, Seo K. Antimicrobial therapy in preterm premature rupture of membranes: results of a prospective, double-blind, placebo-controlled trial of erythromycin. Am J Obstet Gynecol. 1991;165:632-40.

39. Mercer BM, Moretti ML, Prevost RR, Sibai BM. Erythromycin therapy in preterm premature rupture of the membranes: a prospective, randomized trial of 220 patients. Am J Obstet Gynecol. 1992;166:794-802.

40. Cox SM, Leveno KJ, Sherman ML, Travis L, De Plama R. Ruptured membranes at 24 to 29 weeks: a randomized double blind trial of antimicrobials versus placebo. Am J Obstet Gynecol. 1995;172:412.

41. Magwali TL, Cipato T, Majoko F, Rusakaniko S, Mujaji C. Prophylactic Augmentin in prelabour preterm rupture of the membranes. Int J Gynecol Obstet. 1999;65:261-5

42. Kenyon SL, Taylor DJ, Tarnow-Mordi W; ORACLE Collaborative Group. Broad-spectrum antibiotics for preterm, prelabour rupture of fetal membranes: the ORACLE 1 randomised trial. Lancet. 2001;357:979-88.

43. Lewis DF, Adair CD, Robichaux AG, Jaekle RK, Moore JA, Evans AT, et al. Antibiotic therapy in preterm premature rupture of membranes: are seven days necessary? Am J Obstet Gynecol. 2003;188:1413-6.

44. Ernest JM, Givner LB. A prospective, randomized, placebo controlled trial of penicillin in preterm premature rupture of membranes. Am J Obstet Gynecol. 1994;170:516-21.

45. Fuhr NA, Becker C, van Baalen A, Bauer K, Hopp H. Antibiotic therapy for preterm premature rupture of membranes - results of a multicenter study. J Perinat Med. 2006;34:203-6.

46. Kurki T, Hallman M, Zilliacus R, Teramo K, Ylikorkala O. Premature rupture of the membranes; effect of penicillin prophylaxis and long-term outcome of the children. Am J Perinat. 1992;9:11-6.

47. Lockwood CJ, Costigan K, Ghidini A, Wein R, Chien D, Brown BL, et al. Double-blind, placebo-controlled trial of piperacillin prophylaxis in preterm membrane rupture. Am J Obstet Gynecol. 1993;169:970-6.

48. Almeida L, Schmauch A, Bergström S. A randomised study on the impact of peroral amoxicillin in women with prelabour rupture of membranes preterm. Gynecol Obstet Invest. 1996;41:82-4

49. Chatzakis C, Papatheodorou S, Sarafidis K, Dinas K, Makrydimas G, Sotiriadis A. The effect of prophylactic antibiotics for preterm prelabor rupture of membranes on perinatal outcomes: a network meta-analysis of randomized controlled trials. Ultrasound Obstet Gynecol. 2020;55:20-31.

50. Ballinas AH, Farfan JAL, Guevara CG. Comparación de resultados maternos y perinatales en el tratamiento conservador de la rotura prematura de membranas pretérmino entre el uso de eritromicina y clindamicina. Ginecol Obstet Mex. 2011;79:403-10.

51. Kwak H-M, Shin M-Y, Cha H-H, Choi S-J, Lee J-H, Kim J-S, et al. The efficacy of cefazolin plus macrolide (erythromycin or clarithromycin) versus cefazolin alone in neonatal morbidity and placental inflammation for women with preterm premature rupture of membranes. Placenta. 2013:34:346-52.

52. Lovett SM, Weiss JD, Diogo MJ, Williams PT, Garite TJ. A prospective, double-blind, randomized, controlled clinical trial of ampicillin-sulbactam for preterm premature rupture of membranes in women receiving antenatal corticosteroid therapy. Am J Obstet Gynecol. 1997:176:1030-8.

53. American College of Obstetricians and Gynecologists. Practice Bulletins No. 139: Premature rupture of membranes. Obstet Gynecol. 2013;122:918-30.

54. Sociedad Española de Ginecología y Obstetricia. Rotura prematura de membranas. SEGO. Prog Obstet Ginecol. 2012;55:520-40.

55. Royal College of Obstetricians and Gynaecologists. Preterm prelabour rupture of membranes. Green-Top Guideline 44. London: RCOG; 2006. p. 2-12.

56. National Institute for Health and Care Excellence. Preterm labour and birth. NICE guideline. London: NICE; 2015.

57. World Health Organization. WHO recommendations on interventions to improve preterm birth outcome. Geneva: WHO; 2015

58. Yudin $\mathrm{MH}$, van Schalkwyk J, Eyk NV. Antibiotic therapy in preterm premature rupture of the membranes. J Obstet Gynaecol Can. 2009;31:863-7.

59. Guía Perinatal. Subsecretaría de Salud Pública Chile. Programa Nacional Salud de la Mujer. Julio 2015. Capítulo XXII. Rotura prematura de membranas. Pags 291-99.

60. Kenyon S, Pike K, Jones DR, Brocklehurst P, Marlow N, Salt A, et al. Childhood outcomes after prescription of antibiotics to pregnant women with preterm rupture of the membranes: 7-year follow-up of the ORACLE I trial. Lancet. 2008;372:1310-8.

61. Lee JH, Romero R, Kim SM, Chaemsaithong P, Park CW, Park JS, et al. A new anti-microbial combination prolongs the latency period, reduces acute histologic chorioamnionitis as well as funisitis, and improves neonatal outcomes in preterm PROM. J Matern Fetal Neonatal Med. 2016;29:707-20.

62. Grigsby PL, Novy MJ, Sadowsky DW, Morgan TK, Long M, Acosta E, et al. Maternal azithromycin therapy for Ureaplasma intraamniotic infection delays preterm delivery and reduces fetal lung injury in a primate model. Am J Obstet Gynecol. 2012;207:475.e1-14.

63. Ovalle A, Gómez R, Martínez MA, Giglio S, Bianchi R, Poblete P, et al. Antibiotic treatment of patients with term premature rupture of membranes: a randomized clinical trial. Prenat Neonat Med. 1998:3:1-8.

64. Manterola C, Asenjo-Lobos C, Otzen T. Hierarchy of evidence: levels of evidence and grades of recommendation from current use. Rev Chil Infectol. 2014;31:705-18. 